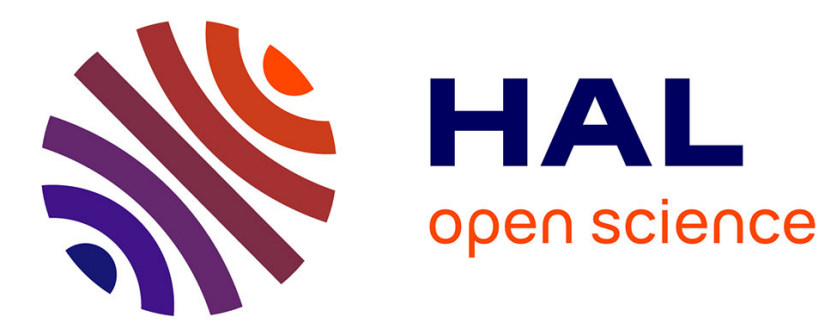

\title{
Multiscale Force Networks in Highly Polydisperse Granular Media
}

Charles Voivret, Farhang Radjai, Jean-Yves Delenne, Moulay Saïd El Youssoufi

\section{To cite this version:}

Charles Voivret, Farhang Radjai, Jean-Yves Delenne, Moulay Saïd El Youssoufi. Multiscale Force Networks in Highly Polydisperse Granular Media. Physical Review Letters, 2009, 102, pp.178001. 10.1103/PhysRevLett.102.178001 . hal-00689872

\section{HAL Id: hal-00689872}

\section{https://hal.science/hal-00689872}

Submitted on 20 Apr 2012

HAL is a multi-disciplinary open access archive for the deposit and dissemination of scientific research documents, whether they are published or not. The documents may come from teaching and research institutions in France or abroad, or from public or private research centers.
L'archive ouverte pluridisciplinaire HAL, est destinée au dépôt et à la diffusion de documents scientifiques de niveau recherche, publiés ou non, émanant des établissements d'enseignement et de recherche français ou étrangers, des laboratoires publics ou privés. 


\title{
Multiscale force networks in highly polydisperse granular media
}

\author{
C. Voivret, F. Radjaï, J.-Y. Delenne and M. S. El Youssoufi \\ LMGC, CNRS-Université Montpellier 2, Place Eugène Bataillon, 34095 Montpellier cedex, France.
}

(Dated: April 20, 2012)

\begin{abstract}
We investigate highly polydisperse packings composed of rigid disks subjected to simple shear by Contact Dynamics simulations. A major unsolved issue is how granular texture and force chains depend on the size polydispersity, and how far they influence the shear strength. Technically, the numerical treatment was made possible by ensuring the statistical representativity of particle size classes with a tractable number of particles. An unexpected finding is that the internal friction angle is independent of polydispersity. Using a Fourier decomposition of the stress tensor, we show that this behavior is related to two mechanisms underlying the stability of force chains: 1) The class of largest particles captures strong force chains, and 2) these chains are equilibrated by weak forces carried by increasingly smaller particles as the size span broadens. Conversely, we find that the Coulomb cohesion in the presence of surface adhesion between particles increases with size polydispersity due to the effect of adhesion on force anisotropy. Our analysis demonstrates the multiscale nature of force networks and its key role for realistic modelling of polydisperse granular materials.
\end{abstract}

PACS numbers: 45.70.-n, 81.05.Rm, 61.43.Hv

Most granular materials occurring in nature and industrial application are composed of a broad range of particle sizes $[1,2]$. Size polydispersity is a key to the space-filling and strength properties of granular materials, and for this reason it needs to be optimized in designing particle-based materials such as concrete [3-5]. However, most research work has focused on model granular systems composed of nearly mono-sized particles. In highly polydisperse granular materials, the particles of a given size can fit into the pores between larger particles. Hence, the space is filled in a hierarchical manner as in apollonian packings [3]. However, unlike apollonian packings in which the fine adjustment of particle positions leads to a fractal structure, the polydisperse granular media are generically disordered and their multiscale microstructure is a consequence of the filling procedure. Novel features are thus expected to emerge due to force transmission through a disordered contact network involving a hierarchy of length scales.

Although size polydispersity is a major characteristic of most granular materials, its impact has not been subject to systematic investigation. The difficulty lies mainly in the preparation of well-calibrated polydisperse samples of the same material in order to be able to isolate properly the effect of polydispersity from other factors such as particle shape, surface effect, etc. On the other hand, a few existing theoretical models have been essentially concerned with solid fraction constructed according to particular space-filling strategies $[2,6-8]$.

In this Letter, we use a numerical simulations to investigate the shear behavior and force transmission in highly polydisperse granular media. We developed a numerical approach allowing us to prepare and shear large packings of circular particles of variable size distribution. The challenge is that a broad size distribution with wellrepresented populations of different sizes requires many more particles than a narrow distribution [9]. In the following, we briefly describe our approach that combines three ingredients: 1) a generic size distribution function, 2) an efficient assembling method and 3) a fast dynamic method for shearing the granular samples.

The size distribution is represented by the cumulate volume distribution defined as the cumulative volume $h(d)$ of the particles as a function of particle diameter $d$. We use a distribution function based on the cumulative $\beta$-distribution which allows us to generate a broad range of distributions with only two parameters controlling the shape of the distribution. The size span is defined by $s=\left(d_{\max }-d_{\min }\right) /\left(d_{\max }+d_{\min }\right)$ where $d_{\min }$ and $d_{\max }$ are the smallest and largest diameters, respectively. A monodisperse distribution corresponds to $s=0$ and the limit $s \simeq 1$ corresponds to an infinitely polydisperse system $[9]$.

For assembling the particles into a dense packing, we use a geometrical deposition method according to simple geometrical rules [10]. Each particle is deposited over those already deposited in the lowest position at the free surface. This method is efficiently implemented in a computer code with periodic boundary conditions in the horizontal direction. The packings prepared by this method are homogeneous in particle size and involve thus no size segregation.

The packings prepared by geometrical procedure are allowed to relax to static equilibrium under a constant vertical stress $\sigma_{v}$ applied on the upper wall. The gravity is set to zero in order to avoid stress gradients. The simulations are performed by means of the contact dynamics method [11, 12], which is the genuine method for the simulation of perfectly rigid particles. We simulated several packings of $10^{4}$ particles with the size span $s$ varying from $s=0.2$ to $s=0.96$. During relaxation the coefficient of friction between particles is set to zero in order to obtain nearly isotropic and dense packings. At the end of relaxation, the solid fraction $\rho$ for each value of $s$ is slightly above its value in the geometrically deposited packing. It varies nonlinearly from $\rho=0.82$ for $s=0.2$ 
to $\rho=0.92$ for $s=0.96$. Two conditions are required to fill efficiently the pores : 1) a broad size distribution, which corresponds to higher values of $s(s>0.4$ in our simulations), 2) a large number of smaller particles, controlled in our model by the shape parameters. These conditions appear to be optimally fulfilled for uniform distribution by volume fractions. A parametric study of the solid fraction as a function of shape parameters shows that the largest value of solid fraction is obtained with uniform distribution by particle volume fraction defined by $h(d)=\left(d-d_{\min }\right) /\left(d_{\max }-d_{\min }\right)$. The above condition appears therefor to be best met for this distribution. The results presented below were obtained with this distribution although they remain valid irrespective of shape parameters. Given the disordered microstructure of these samples with an optimal filling of space, they can be qualified as polydisperse random close packings.

The coefficient of friction between particles is set to $\mu=0.4$ in the relaxed packings and they are sheared by subjecting the upper wall to a small horizontal velocity $v$ and constant confining pressure $\sigma_{v}$ while the lower wall is kept immobile. During shear, all samples dilate from their initially high density and tend to a constant solid fraction in the steady state depending on $s$. The stress tensor in the sample is given by $\sigma_{i j}=n_{c}\left\langle f_{i}^{c} \ell_{j}^{c}\right\rangle$ where $n_{c}$ is the number density of contact and the average is taken over the contacts $c$ with contact force $f^{c}$ and branch vector $\ell^{c}$ joining the centers of contacting particles $[13-16,18]$. The mean stress is $p=\left(\sigma_{1}+\sigma_{2}\right) / 2$ where $\sigma_{1}$ and $\sigma_{2}$ are the principal stress values. We also define a stress deviator $q=\left(\sigma_{1}-\sigma_{2}\right) / 2$. During shear, the shear stress jumps initially to a high value before decreasing to a nearly constant value in the steady state. The steadystate shear stress $q^{*} / p$ characterizes the shear strength of the material. According to the Mohr-Coulomb model, the shear strength of granular materials can be split in two contributions: 1) the internal angle of friction $\varphi$ and 2 ) the Coulomb cohesion $c$. In 2D, these two parameters are related to $q^{*}$ by [1]

$$
q^{*}=p \sin \varphi^{*}+c \cos \varphi^{*} .
$$

Figure 1 shows $\varphi^{*}$ as a function of $s$ for cohesionless packings. Hence, according to Fig. $1, \varphi^{*}$ is almost independent of size span $s$. Surprisingly, we observe that $\varphi^{*}$ is almost independent of size $\operatorname{span} s$. This result is rather counter-intuitive as it is often believed that the shear strength in granular materials should increase with solid fraction, which is an increasing function of $s$. In order to understand the origin of this paradox we analyze in the following the microstructure and force transmission.

Figure 2(a) displays a snapshot of the force network for $s=0.96$. We observe strong force chains preferentially passing through larger particles. At the same time, a large number of small particles are excluded form the force network. In other words, the large particles capture strongest force chains whereas small particles often miss them. The packing becomes therefore more inhomogeneous in force transmission as the size span becomes

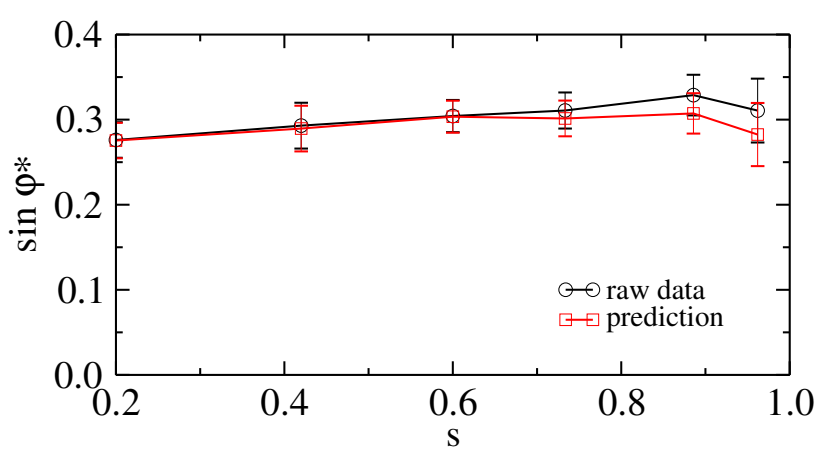

FIG. 1: Internal friction $\sin \varphi^{*}$ as a function of particle size span $s$ both from raw simulation data and as predicted by Eq. 5. The error bars indicate the standard deviation of stress fluctuations in the steady state.

broader. This is best illustrated by the probability density function (pdf) of normal forces shown in Fig. 3 for different values of $s$ [13]. We see that the pdf becomes broader as $s$ increases, and the population of weak forces (below the mean force) grows significantly as a function of $s$. This enhanced force inhomogeneity is in strong contrast with the fact that the packing becomes more homogeneous in terms of solid fraction as the size span broadens.

The force network is linked with the stress components via the expression of stress tensor that we write down here in its integral form [13-16]:

$$
\sigma_{\alpha \beta}=n_{c} \iiint f_{\alpha}(\vec{n}) \ell_{\beta}(\vec{n}) P_{f \ell n}(\vec{f}, \ell, \vec{n}) d \vec{f} d \ell d \vec{n}
$$

where $P_{f \ell n}(\vec{f}, \ell, \vec{n})$ is the joint pdf of the forces $\vec{f}$ and branch vectors $\vec{\ell}=\ell \vec{n}$. At the lowest-order description of the force network, we neglect the correlations and split the joint probability function as a product of three independent functions $P_{f \ell n}(\vec{f}, \ell, \vec{n})=P_{f}(\vec{f}) P_{\ell}(\ell) P_{n}(\vec{n})$. Then, integration over $\vec{f}$ and $\ell$ yields

$$
\sigma_{\alpha \beta} \simeq n_{c} \int_{\Omega}\left\langle f_{\alpha}\right\rangle(\vec{n})\left\langle\ell_{\beta}\right\rangle(\vec{n}) P_{n}(\vec{n}) d \vec{n}
$$

where $\Omega$ is the angular domain of integration, and $\langle\ell\rangle(\vec{n})$ and $\langle f\rangle(\vec{n})$ are the average branch vector length and force as a function of contact orientation vector $\vec{n} \equiv$ $(\cos \theta, \sin \theta)$ in $2 \mathrm{D}$. In this framework, a model of stress transmission is reduced to the choice of the functions $\langle\ell\rangle(\theta),\langle\vec{f}\rangle(\theta)$ and $P_{n}(\theta)$. The contact force $\vec{f}$ can be represented by its normal and tangential components $f_{n}(\theta)$ and $f_{t}(\theta)$.

The above four functions describe the general state of the packing. However, under shearing the packing self-organizes itself into a unimodal state where they are well approximated by their lowest-order Fourier expan- 


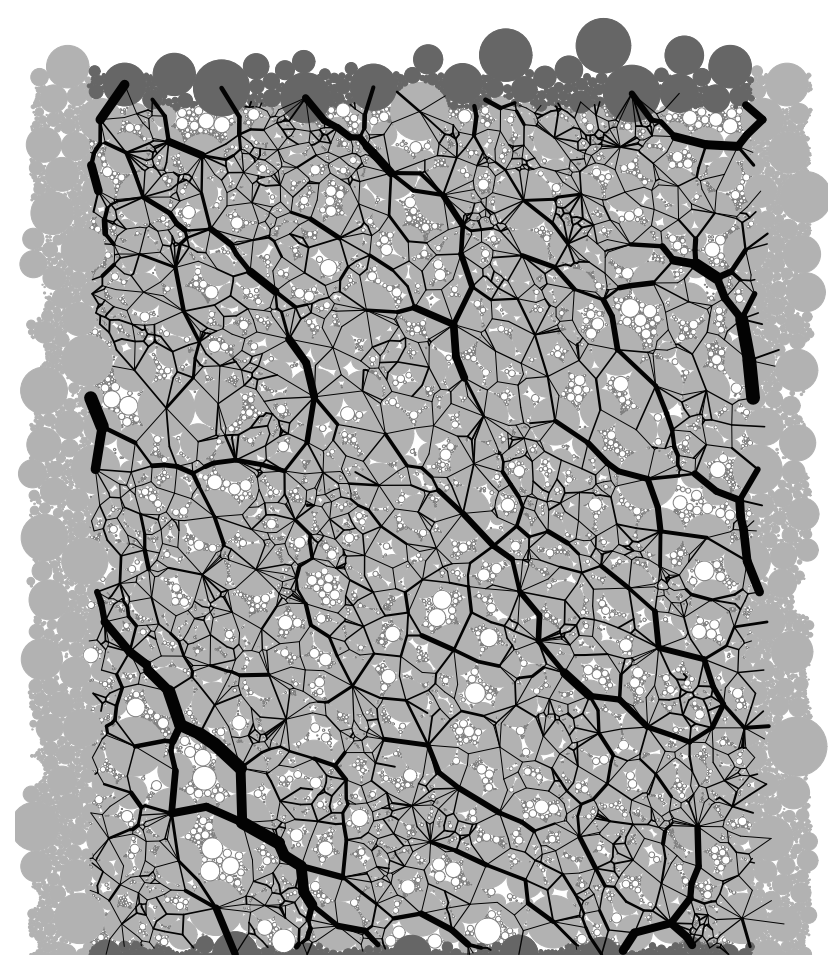

(a)

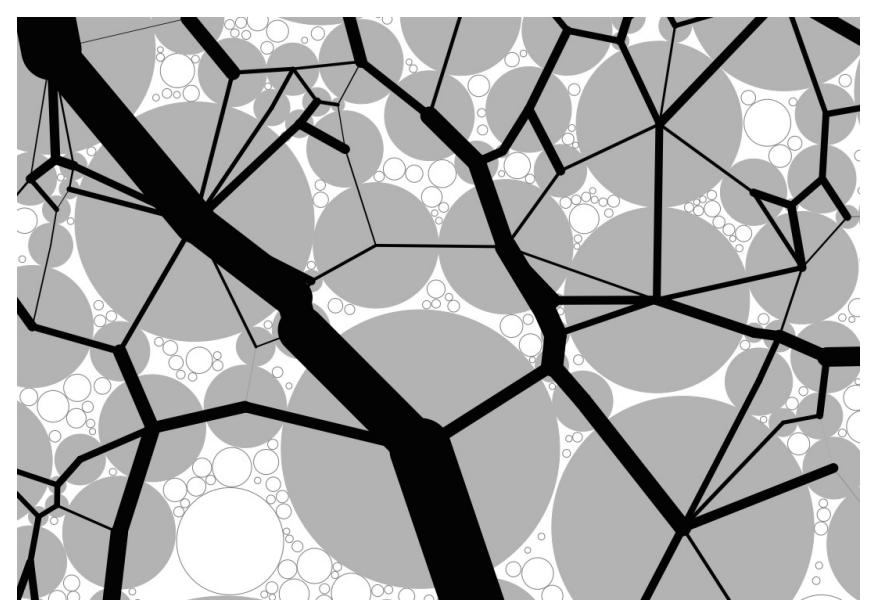

(b)

FIG. 2: A snapshot of a highly polydisperse packing with size span $s=0.96$. The floating particles, excluded from the force network are in white. Line thickness is proportional to normal force.

sion $[16,17,19]$ :

$$
\left\{\begin{array}{l}
P_{n}(\theta) \simeq \frac{1}{\pi}\left\{1+a_{c} \cos 2\left(\theta-\theta_{c}\right)\right\} \\
\langle\ell\rangle(\theta) \simeq\langle\ell\rangle\left\{1+a_{\ell} \cos 2\left(\theta-\theta_{\ell}\right)\right\} \\
\left\langle f_{n}\right\rangle(\theta) \simeq\left\langle f_{n}\right\rangle\left\{1+a_{n} \cos 2\left(\theta-\theta_{n}\right)\right\} \\
\left\langle f_{t}\right\rangle(\theta) \simeq-\left\langle f_{n}\right\rangle a_{t} \sin 2\left(\theta-\theta_{t}\right)
\end{array}\right.
$$

where $a_{c}, a_{\ell}, a_{n}$ and $a_{t}$ are anisotropy parameters and

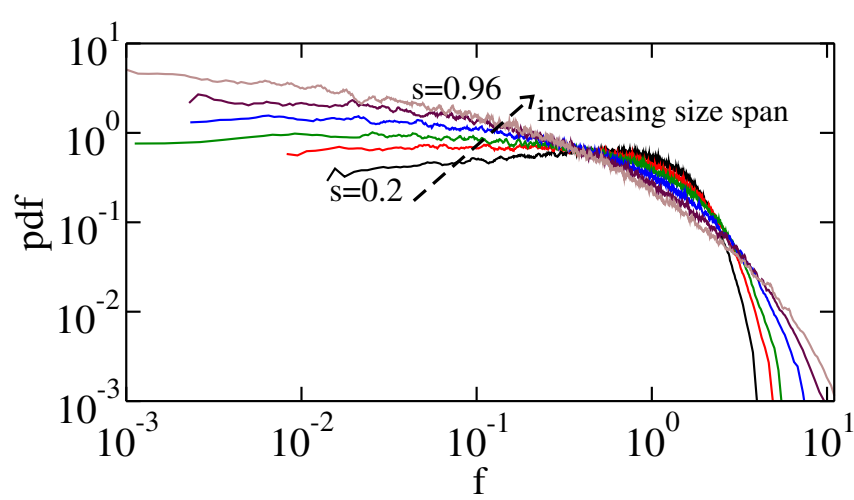

FIG. 3: Probability distribution function of normal forces $f=f_{n} /\left\langle f_{n}\right\rangle$ normalized by the average force $\left\langle f_{n}\right\rangle$ for different values of size span $s$.

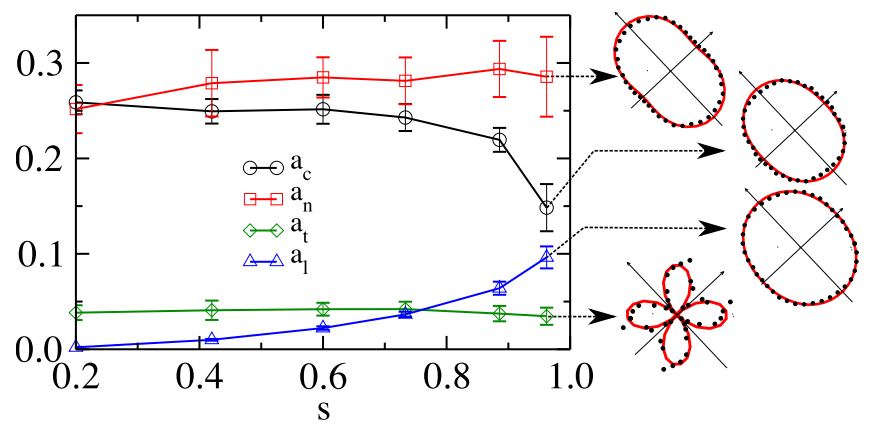

FIG. 4: Evolution of the anisotropy parameters (see text) as a function of size span in the steady shear state. The error bars correspond to the standard deviation of the fluctuations in the steady state. The polar diagrams of the corresponding angular distributions (black symbols) are shown for $s=0.96$ together with theirs fits (red) by truncated Fourier expansions (Eq. 4)

the angles $\theta_{c}, \theta_{\ell}, \theta_{n}$ and $\theta_{t}$ represent the corresponding privileged directions; see figure 4 . In general, the privileged angles can be all different, but in a sheared state they tend to follow the principal stress direction $\left(\theta_{\sigma}=\theta_{c}=\theta_{\ell}=\theta_{n}=\theta_{t}\right)[19,20]$. Now, inserting the Fourier expansions (4) into the integral expression (3) of stress and neglecting cross products between the anisotropy parameters, one gets

$$
\sin \varphi^{*}=\frac{q^{*}}{p} \simeq \frac{1}{2}\left(a_{c}+a_{\ell}+a_{n}+a_{t}\right) .
$$

The predicted values of $\sin \varphi^{*}$ by this equation are shown in figure 1 together with the measured values as a function of $s$. We see that equation (5) approximates well the friction angle for all values of $s$.

The evolution of the four anisotropies with $s$ is displayed in Fig. 4 from the simulation data. Interestingly, the force anisotropies $a_{n}$ and $a_{t}$ are independent of $s$. This is consistent with the observation that the strong force chains, underlying the force anisotropies for the most part, are mainly guided by the class of largest particles irrespective of $s$. However, the contact orientation 


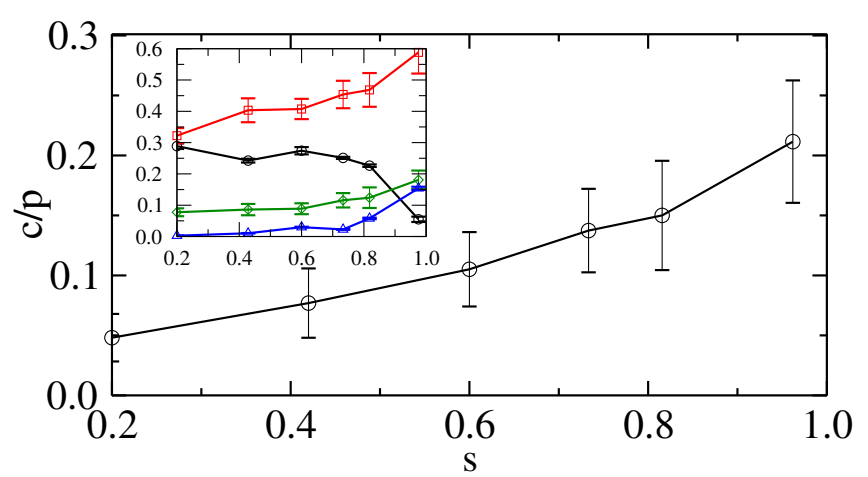

FIG. 5: Evolution of Coulomb cohesion $c$ normalized by the average pressure in the steady state as a function of size span $s$. The error bars correspond to the standard deviation of stress fluctuations in the steady state. The inset shows the steady-state anisotropies as a function of $s$.

anisotropy $a_{c}$ declines with $s$, reflecting the fact that the larger particles are surrounded by an increasing number of small particles as $s$ increases. At the same time, the length anisotropy $a_{\ell}$ increases with $s$ since the longest branch vectors occur between largest particles that align themselves with the strong force chains. It is remarkable that $a_{c}$ and $a_{\ell}$ evolve with $s$ but their sum $a_{c}+a_{\ell}$ remains constant. By virtue of Eq. 5, this compensation between $a_{c}$ and $a_{l}$, together with the fact that $a_{n}$ and $a_{t}$ do not evolve with $s$, implies that $\varphi^{*}$ is independent of size span. In this way, the independence of the internal friction angle with respect to size polydispersity appears to be a consequence of the multiscale nature of the force network. Schematically, the force chains cascade from large scales (large particles) down to small scales (small particles), and the effect of increasing size span is to replace the particles propping the strong force chains by a growing population of smaller particles.

In order to elucidate the effect of polydispersity in the presence of cohesion, we performed simple shear simulations under the same boundary conditions as before but with an additional constant adhesion force $-f_{0}$ between contacting particles. The adhesion force being reversible, we obtain cohesive packings with well-defined shear strength in the steady state. We find that the shear strength $q^{*} / p$ increases with $s$ whereas $\varphi^{*}$ keeps the same value as in cohesionless packings. Hence, according to Eq. (1), the Coulomb cohesion $c$ increases with size span $s$ as shown in Fig. 5. The inset shows the steady-state anisotropies as a function of $s$. We see that the main contribution to cohesion stems from the force anisotropies $a_{n}$ and $a_{t}$ that increase with $s$ given the same constant value of local adhesion. In other words, the effect of adhesion is enhanced by size polydispersity.

In summary, our simulations provide clear evidence that in highly polydisperse granular media, the internal friction angle is independent with respect to particle size span. This unexpected feature was shown to be a consequence of the interplay between force chains and a hierarchy of length scales. Our results also clarify the amplifying effect of polydispersity on the Coulomb cohesion of cohesive granular materials.

These findings are relevant to a wide class of granular materials where size polydispersity and adhesion are essential to the behavior. Since the force networks present generally similar phenomenology in $2 \mathrm{D}$ and $3 \mathrm{D}$, we expect that our results will apply also in 3D. Presently, 3D simulations with highly polydisperse granular packings of spheres are under way, but require considerably more particles for the sake of statistical representativity of size populations and thus much more demanding computation time.
[1] J. K. Mitchell and K. Soga, Fundamentals of Soil Behavior, third edition (Wiley, 2005).

[2] T. Aste and D. Weaire., The Pursuit of Perfect Packing (Institute of Physics Publishing, Bristol and Philadelphia, 2000).

[3] H. J. Herrmann, R. Mahmoodi Baram, and M. Wackenhut, Physica A: Statistical Mechanics and its Applications 330, 77 (2003).

[4] F. de Larrard, Concrete Mixture Proportioning (E \& FN Spon, 1999).

[5] H. J. Herrmann, J. A. Astrom, and R. Mahmoodi Baram, Physica A: Statistical Mechanics and its Applications 344, 516 (2004).

[6] A. Gervois, C. Annic, J. Lemaitre, M. Ammi, L. Oger, and J. P. Troadec, Physica A: Statistical and Theoretical Physics 218, 403 (1995).

[7] A. Gervois and D. Bideau, in Disorder and Granular Media, edited by D. Bideau (Elsevier, Amsterdam, 1993).

[8] P. Richard, L. Oger, J.-P. Troadec, and A. Gervois, Phys. Rev. E 60, 4551 (1999).
[9] C. Voivret, F. Radjai, J.-Y. Delenne, and M. S. El Youssoufi, Phys. Rev. E 76, 021301 (2007).

[10] W. M. Visscher and M. Bolstreli, Nature 239, 504 (1972).

[11] J. Moreau, European Journal of Mechanics A/Solids supp., 93 (1994).

[12] F. Radjai, European Journal of Environmental and Civil Engineering 12, 871 (2008).

[13] F. Radjai, D. E. Wolf, M. Jean, and J.-J. Moreau, Phys. Rev. Lett. 80, 61 (1998).

[14] M. M. Mehrabadi, S. Nemat-Nasser, and M. Oda, Int. J. Num. Anal. Meth. Geomech. 6, 95 (1982).

[15] J. Christoffersen, M. M. Mehrabadi, and S. NematNasser, J. Appl. Mech. 48, 339 (1981).

[16] L. Rothenburg and R. J. Bathurst, Geotechnique 39, 601 (1989).

[17] B. Cambou, in Powders and Grains 93, edited by C. Thornton (A. A. Balkema, Amsterdam, 1993), pp. 73-86.

[18] W.G. Ellenbroek and J.H. Snoeijer, J. Stat. Mech. , P01023 (2007). 
[19] E. Azéma and F. Radjai and G. Saussine, Mechanics of Materials , 10.1016/j.mechmat.2009.01.021 (2009).

[20] L. Rothenburg and R.J. Bathurst and A.A. Berlin, in
Powders and Grains 93, edited by C. Thornton (A. A. Balkema, Amsterdam, 1993), pp. 147-153. 